\title{
Restoration of Thickness, Density, and Volume for Highly Blurred Thin Cortical Bones in Clinical CT Images
}

\author{
Amirreza Pakdel, Michael Hardisty, Jeffrey Fialkov, \\ and Cari Whyne
}

Version Post-Print/Accepted Manuscript

Citation Pakdel A, Hardisty M, Fialkov J, Whyne C. Restoration of Thickness, (published version) Density, and Volume for Highly Blurred Thin Cortical Bones in Clinical CT Images. Ann Biomed Eng. 2016 May 31. doi: 10.1007/s10439-0161654-y. [Epub ahead of print]. PMID: 27245852.

Publisher's Statement The final published version of this article is available at Springer Verlag via https://dx.doi.org/10.1007/s10439-016-1654-y.

Always cite the published version, so the author(s) will receive recognition through services that track citation counts, e.g. Scopus. If you need to cite the page number of the TSpace version (original manuscript or accepted manuscript) because you cannot access the published version, then cite the TSpace version in addition to the published version using the permanent URI (handle) found on the record page. 


\title{
Restoration of thickness, density, and volume for highly blurred thin cortical bones in clinical CT images
}

\author{
Amirreza Pakdela, Michael Hardistyb, Jeffrey Fialkovb,d, Cari Whyne a,b,c \\ anstitute of Biomaterials and Biomedical Engineering, University of Toronto, Toronto, \\ ON, Canada \\ ${ }^{b}$ Sunnybrook Health Sciences Center, Toronto, ON, Canada \\ ${ }^{c}$ Faculty of Medicine, University of Toronto, Toronto, ON, Canada dDivision of Plastic and Reconstructive \\ Surgery, University of Toronto, Toronto, ON, Canada
}

This is the Final Manuscript. Published as: Restoration of Thickness, Density, and Volume for Highly Blurred Thin Cortical Bones in Clinical CT Images. Pakdel A, Hardisty M, Fialkov J, Whyne C. Ann Biomed Eng (2016) 44:3359-3371 DOI 10.1007/s10439-016-1654-y The final publication is available at link.springer.com.

\begin{abstract}
In clinical CT images containing thin osseous structures, accurate definition of the geometry and density is limited by the scanner's resolution and radiation dose. This study presents and validates a practical methodology for restoring information about thin bone structure by volumetric deblurring of images. The methodology involves 2 steps: a phantom-free, post-reconstruction estimation of the 3D point spread function (PSF) from CT data sets, followed by iterative deconvolution using the PSF estimate. Performance of 5 iterative deconvolution algorithms, blind, Richardson-Lucy (standard, plus Total Variation versions), Modified Residual Norm Steepest Descent (MRNSD), and Conjugate Gradient Least-Squares were evaluated using CT scans of synthetic cortical bone phantoms. The MRNSD algorithm resulted in the highest relative deblurring performance as assessed by a cortical bone thickness error $(0.18 \mathrm{~mm})$ and intensity error $(150 \mathrm{HU})$, and was subsequently applied on a CT image of a cadaveric skull. Performance was compared against micro-CT images of the excised thin cortical bone samples from the skull (average thickness $1.08 \pm 0.77 \mathrm{~mm}$ ). Error in quantitative measurements made from the deblurred images was reduced $82 \%(p<0.01)$ for cortical thickness and 55\% $(p<0.01)$ for bone mineral mass. These results demonstrate a significant restoration of geometrical and radiological density information derived for thin osseous features.
\end{abstract}


Key terms: quantitative image analysis, computed tomography (CT), cortical thickness and intensity, point spread function, deconvolution

\section{Introduction}

X-ray computed tomography (CT) is the primary modality for three-dimensional (3D) imaging of bony tissue and construction of anatomical models of bone $e^{6,12,19,30}$. Voxels associated with bony structures have high Hounsfield unit (HU) intensity compared to other anatomical structures which enables visualization and segmentation. However, the resolution of clinical CT images can limit visualization and segmentation of thin bone structures.

The bones of the craniomaxillofacial skeleton (CMFS), particularly those around the orbit and sinus that encapsulate the maxillary and paranasal cavities are the thinnest in the human body $^{1,45}$, with a thickness (approximately $0.4-1.5 \mathrm{~mm}$ ) of a similar size to the spatial resolution achievable with many clinical CT imaging systems. As such, measurements of the thickness and intensity in these thin bones are dominated by the behaviour of the imaging system, which can result in severe overestimation of thickness and underestimation of intensity magnitude ${ }^{17,20,24,40}$.

The point-spread function (PSF) of an imaging system characterizes the size of the smallest details that may be resolved in an image. A general form of an imaging system is:

$$
f=K f_{\text {true }}+\epsilon,
$$

where $f$ is the discretized representation of the observed image and $f_{\text {true }}$ is the non-deblurred and noise-free idealized discretization of the object being imaged. $K$ is the kernel matrix defined by the imaging system's PSF, and $\varepsilon$ is the error due to noise. The objective of deblurring is to recover $f_{\text {true }}$ given $f$ and $K^{4,10}$. The challenge is that the frequency bandwidth of the random noise in the image extends well beyond the high frequency components of the PSF. Attempting to solve for $f_{\text {true }}$ by direct deconvolution can amplify higher frequency noise to unacceptable levels. Finding an acceptable solution for $f_{\text {true }}$ involves regularization, the process of screening the high frequency information for what might have a high probability of being details representing $f_{\text {true }}$, while rejecting those that are more likely to be noise. Numerous iterative deconvolution algorithms have been developed to provide an optimized estimation of $f_{\text {true }}$ through various strategies such as conjugate gradients least squares, or statistically based schemes such as expectation-maximization. While $f_{\text {true }}$ cannot be perfectly recovered, an estimation that is superior to $f$ is possible. 
The PSF of a reconstructed CT image depends on numerous scanning parameters, such as imaging system hardware, specific protocol and reconstruction kernel. Thus, when presented with images obtained from a CT scanner, the PSF is generally unknown and can be difficult to obtain. It is possible to estimate the PSF, however, from parts of a CT image exhibiting abrupt changes in intensity (i.e. contrast agents within vessels or bone-tissue interfaces), without additional scanning or modification of protocols to include reference markers or phantoms ${ }^{16,27}$. This method can be used on any previously-acquired CT image data or on data sourced from a clinical scanner that may not be directly accessible. The estimated PSF can then be supplied to iterative deconvolution algorithms to restore the entire CT volume. 3D reconstructions of synthetic anatomies produced from deconvolved CT stacks have been shown to contribute to significant accuracy gains ${ }^{31}$; however, this has not been previously demonstrated or optimized in human models, where the contrast-resolution of thin cortical bone regions is greatly diminished by the surrounding soft tissue.

This article presents a methodology for improving thin bone geometry and intensity information in human CMFS CT images by iterative deconvolution methods that use an estimated Point-Spread Function (PSF). This investigation has been motivated by the need for greater accuracy in the generation of patient specific CMFS models used for skeletal biomechanical studies, which heavily rely on accurate geometrical representation of objects, as well as precise localization of material properties ${ }^{11,41,47}$. Further, a practical method for restoring information in CT has potentially wide-ranging clinical applications, such as improving baseline diagnostic sensitivity, increasing the reliability of information used in pre-operative planning, and enabling better contour conformity and compatibility in the design of patient-specific implants $^{23,31}$.

\section{Materials and Methods}

In this investigation image restoration was performed on bone mimicking phantoms of known geometry with 5 different deconvolution algorithms to evaluate their performance. The algorithm with the best overall performance was then applied to cadaveric CT images of the CMFS and evaluated with respect to thickness and intensity measurements from excised bone fragments imaged using a micro CT scanner. 


\section{PSF estimation}

The authors have previously described a process for 3D PSF estimation in CT images which contain thin cortical bones ${ }^{27}$. Briefly the main steps of this method are: 1) Identification of cortical bone regions in the CT, which are sufficiently thin such that they can be assumed to have a near constant density through their thickness and are sufficiently flat such they can be assumed planar; 2) Extraction of intensity profiles along lines normal to the surface of these thin cortical bones (Figure 1). Note that profiles were found both within the transverse plane and at differing tilt angles $(\theta)$ with the transverse CT slices; 3) Numerical solution of the model (assumed to be "box car" functions, blurred by the system's PSF, (equation 2) to estimate the "unblurred" intensity profile and the PSF using a non-linear interior point optimization solver (MATLAB, Mathworks Inc., Natick, MA).

The blurring of the cortical bone's intensity profile $(\mathrm{g})$ is modelled $^{27}$ by the convolution of a rectangular function with the unknown 3D PSF (assumed 3D Gaussian, radially symmetric within the transverse plane) to be solved for by the expression:

$$
g\left(x^{\prime}, y^{\prime}\right)=\frac{Y_{2}}{\sqrt{2 \pi} \sigma_{r}} \int_{-\frac{W}{2}}^{\frac{W}{2}} e^{-\frac{\left(x^{\prime}-\tau_{1}\right)^{2}}{2 \sigma_{r}^{2}}} d \tau_{1}=\frac{Y_{2}}{2}\left[\operatorname{erf}\left(\frac{x^{\prime}+\frac{W}{2}}{\sigma_{r} \sqrt{2}}\right)-\operatorname{erf}\left(\frac{x^{\prime}-\frac{W}{2}}{\sigma_{r} \sqrt{2}}\right)\right]
$$

Where $\sigma_{r}=\sqrt{\sigma_{x y}^{2} \cos ^{2} \theta+\sigma_{z}^{2} \sin ^{2} \theta}$.

The intensity of the cortical bone is given by the unknown variable $Y_{2}$. W is the unknown width of the bone, spanning the interval from $X_{1}$ to $X_{2}$ (Figure 1). The parameters $\sigma_{x y}$ and $\sigma_{z}$ are the standard deviations of the PSF in the transverse plane and axial directions. $\theta$ is the tilt angle between the normal of the bone surface and the transverse plane of the CT volume. The PSF is estimated by iterative non-linear fitting of equation 2 along multiple profiles to obtain the unknowns (Y2, W, $\left.\sigma_{x y}, \sigma_{z}\right)$ as previously validated ${ }^{27,29}$. The PSF once obtained from the optimization was 3D. The shape of the PSF assumed within this work resulted in different amounts of blurring depending on the angle $\theta$ as described by $\sigma_{r}$. 


\section{Deconvolution algorithms}

Five deconvolution algorithms (Table 1) were investigated. The Richardson-Lucy (RL) iterative deconvolution algorithm was utilized as it has been extensively studied ${ }^{2,4,7,37,42}$. An implementation of RL that includes regularization by Total Variation (RL-TV) was chosen as it adds the option of specifying a regularization parameter $(\lambda)$ which influences noise in the deblurred image. The RL-TV was applied and evaluated at four different $\lambda$ levels which enables a determination of the importance of regularization. The conjugate-gradient least squares (CGLS) and the modified residual norm steepest descent (MRNSD) methods were chosen as they have demonstrated superior performance to RL in the deblurring of 2-D images ${ }^{3}$. Finally, the Blind algorithm does not require the input of PSF, and attempts to deblur the image and estimate the PSF simultaneously. This method was included as a reference point for a measure of restoration which does not require a priori PSF knowledge. Existing implementation of these algorithms in MATLAB (Mathworks Inc., Natick, MA) or ImageJ ${ }^{34}$ were used.

\section{Phantom experiment}

Six phantom configurations were scanned and processed (Table 2). The phantoms were designed to mimic a thin single cortical layer as well as bi-cortical sandwich structures found in shell bones (CMFS, pelvis, and scapula). Layers of Teflon and Delrin were used as CT radiological equivalent materials for simulation of cortical and trabecular bone, respectively, as they match the $\mathrm{HU}$ intensity of these tissues in the diagnostic ranges of x-ray considered. The layers were sandwiched and vacuum compressed within water equivalent materials (Figure 2). The sheet layers were aligned parallel to the coronal plane and at a tilt angle of $\theta=35^{\circ}$ away from the coronal plane (Table 2).

Phantoms were scanned in a GE Lightspeed VCT (GE Health Care, Waukesha, WI) at 120 $\mathrm{kV}$ and $300 \mathrm{~mA}$ tube voltage and current, and reconstructed using a standard kernel to a $0.48 \times 0.48 \times 0.6 \mathrm{~mm}$ voxel size, (manufacturer's reported FWHM 0.7mm). The PSF for each of the image sets described below were estimated independently, using the method described above. A minimum of 20 intensity profiles were extracted for each image set, and the mean $\sigma_{x y}$ and $\sigma_{z}$ were used to construct a parametric 3D Gaussian PSF for each image. The PSF was discretized such that the PSF voxel size conformed to those of the image volume. 
Scans were resampled to create isotropic voxels $\left(0.1 \times 0.1 \times 0.1 \mathrm{~mm}^{3}\right)$ with a Lanczos kernel ${ }^{21}$ (Amira, Visage Imaging, San Diego, CA). Each phantom image was deblurred by the five algorithms listed in Table 1, for 25, 50, 75, 100 and 150 iterations. Prior to deblurring, image borders were zero padded to minimize the induction of ringing artifacts (Gibbs oscillations) by the deconvolution process.

To measure the quality of the deconvolution methods cortical feature thickness, intensity and intensity variance were determined and compared with known values. The average thickness of the cortical feature and their standard deviations were determined by segmentation (described below) of the CT volumes. The thickness of the segmentations was compared to the true thickness of the Teflon layers as measured directly with a digital caliper. The mean and variance of the intensity of the voxels contained within the segmentation boundaries was also calculated. The mean was compared to the theoretical value of Teflon's intensity in calibrated clinical CT images to determine the absolute intensity error. Figure 3 visually depicts the three criteria variables described above using an exemplary intensity profile through layers of a phantom. The variance of the voxel intensities was calculated to quantify homogeneity of the density (intensity), which is influenced by factors such as noise and partial volume.

Automatic connected components segmentation was used to construct the 3D surface geometry of the cortical (Teflon) layers, using four sequential intensity threshold levels. The highest threshold (709 Hounsfield units (HU)) was chosen as the expected half-way intensity between Teflon and Delrin (1000 and 418 HU, respectively). This approximates the ideal fullwidth half-max (FWHM) value, at which the segmentation of objects with minimal blurring accurately captures their geometric boundaries ${ }^{32}$. The other threshold levels were set at equal intervals between 709 and 418 HU. After automatic segmentation, a binary voxel image mask representing the extracted geometry was created. An Euclidian Distance Transform (EDT) was then applied, and separate binary images containing only the boundary edges and the center line of the segmentations were generated using binary morphological operations (erosion by a cubic matrix of 1's, and skeletonization). Thickness and intensity errors within each scan was typically based on > 30,000 and > 200,000 samples, respectively, with variability depending on segmentation threshold and the size of the segmented volume. 
Iterative deconvolution algorithms exhibit semi-convergence behaviour ${ }^{43}$. Described briefly, this behaviour means that each iteration increases the quality of the restoration, but only to a minimum, which is the point of semi-convergence. Beyond this, each new iteration propagates noise errors, thus decreasing the signal-to-noise ratio (SNR) ratio without offering any further real restoration. The ideal number of iterations to semi-convergence was determined by fitting the improvements in thickness error, intensity error and intensity variance observed for each algorithm at the observed iterations $(25,50,75,100,150)$. All three quality criteria were given equal weight. The goal was set to determine the number of iterations that offered the highest reduction in the thickness and intensity error while minimizing increases in the intensity variance. Since each deconvolution iteration amplifies noise to a degree, the intensity variance calculated for the non-deblurred data sets was chosen as the lowest variance reference (variance desirability = 1). Conversely, each iteration is expected to reduce the thickness and intensity errors. Therefore, the thickness and intensity errors calculated in the non-deblurred data set were set as the highest error reference (thickness and intensity error desirability $=0$ ).

The number of iterations towards optimal convergence and the overall computational time for the algorithms were not used as performance metrics due to the disparity in the utilization of multi-CPU parallel processing and GPU resources by the different deconvolution implementations in MATLAB and ImageJ software. All deconvolutions were performed on a workstation powered by dual $2.5 \mathrm{GHz}$ Xeon CPUs (6-cores each, 15MB L3 cache) and 32GB of DDR3 memory.

Pairwise t-tests were used to determine significant differences in performance between the algorithms and original scan (JMP, SAS, Cary, NC, USA). Differences in thickness and intensity errors as well as intensity standard deviation were examined. The experimental unit considered was the mean result from each deconvolved algorithm at a specific threshold. The effect of threshold on thickness and intensity errors was not the focus of this investigation and has been previously studied ${ }^{40}$. All thresholds used were pooled in the pair-wise t-test.

\section{CMFS cadaveric experiment}

A fresh-frozen cadaveric male human head, disjoined at the fourth cervical vertebrae, was scanned on a GE Lightspeed VCT and reconstructed to $0.48 \times 0.48 \times 1.00 \mathrm{~mm}$ voxel size. The scanner parameters were a pre-set clinical CMFS protocol, reconstructed with the "standard" 
filter. Prior to deblurring, the image's region of interest (ROI) was cropped to exclude as much of the surrounding air volume as possible, to reduce unnecessary computational burden for deconvolution. The cropped image matrix maintained a uniform air intensity field in five of the six boundaries of the image matrix, mollifying the potential for generating edge ringing artifacts through deconvolution. The image was resampled to an isotropic resolution of $0.25 \times 0.25 \times 0.25$ $\mathrm{mm}$.

Following the clinical CT scan of the specimen, five fragments were excised from maxillary, zygomatic, and nasal regions (Figure 4). These samples were composed of cortical bone entirely, or at a minimum, sub-mm to $<2 \mathrm{~mm}$ thick cortical bone constituted $90 \%$ of their volume. Each fragment was scanned at a resolution of $13.7 \times 13.7 \times 13.7 \mu \mathrm{m}$ voxel size on a GE Explore Locus (GE Health Care, Waukesha, WI). Bone mineral density phantoms composed of hydroxyapatite were included with each scanned image for calibration of the voxel intensity values to bone ash density.

Each $\mu \mathrm{CT}$ image was registered onto its originating physical location on the clinical CT image by manual registration, followed by fine-tuned registration using automatic affine registration (Amira 5.2, FEI, Hillsboro, OR, USA). Location landmarks from a 3-D spatial coordinate digitizer (MicroScribe, Immersion Corp., San Jose, CA, USA) ensured accuracy of the 3-D registrations, despite the large difference in resolution scales (Figure 4). The $\mu \mathrm{CT}$ 's image matrix was transformed and mapped onto the clinical CT Cartesian coordinate system by 3 -D resampling. This was followed by further sampling to $0.05 \times 0.05 \times 0.05 \mathrm{~mm}$ voxel size to reduce the image size for the post-processing and 3-D volume visualization operations. It was verified that there was no significant difference between the geometrical volume, thickness and intensity distribution of the down-sampled cortical volumes and that of the original $\mu \mathrm{CT}$ data set after resampling. The original and the deblurred clinical CT images were likewise resampled to $0.05 \times 0.05 \times 0.05 \mathrm{~mm}$ resolution in order to establish a coherent voxel coordinate system for comparison to the $\mu \mathrm{CT}$ reference data (Figure 5).

The deblurred clinical CT volume was compared against $\mu \mathrm{CT}$. True cortical thickness and density in the cadaveric specimen were measured from $\mu \mathrm{CT}$ images. Cortical bone regions in the $\mu \mathrm{CT}$ scans were manually segmented. From the segmentation boundaries, the $3 \mathrm{D}$ volumes were extracted as high resolution tetrahedral mesh surfaces. The mean and standard deviation of the thickness for each $\mu \mathrm{CT}$ cortical volume were calculated at all of its mesh surface vertices, 
totalling approximately $>300,000$ thickness measurements for each sample. The volume of the segmented geometry and the mean and standard deviation of the voxel intensity values distribution within the cortical segmentation boundaries were also calculated. The $\mu \mathrm{CT}$ voxel intensity values were converted into bone ash density using a linear calibration scale established through scanning of hydroxyapatite phantoms. The "radiological mass" (in grams) of the cortical regions in the $\mu \mathrm{CT}$ images were then determined from the numerical integral of the mass of individual voxels based on their associated intensity-derived density and their cubic volumes. Radiological mass captures both the volumetric accuracy and the accuracy of the density calculated for the segmented region.

Five sub-volumes of the original $\mathrm{CT}$ image $\left(\mathrm{CT}_{0}\right)$ and the deblurred results $\left(\mathrm{CT}_{\text {deblurred }}\right)$ were generated by cropping to the ROI of their respective $\mu \mathrm{CT}$ reference regions. Zero-padding was applied to any voxel in the cropped volumes that lay adjacent to the excision boundaries of the $\mu \mathrm{CT} 3 \mathrm{D}$ surfaces. The cortical regions of the $\mathrm{CT}_{0}$ and $\mathrm{CT}_{\text {deblurred }}$ images were segmented by automatic threshold segmentation at three different minimum intensity thresholds, which were individualized for each region based on the mean $\left(T_{\mu C T}\right)$ and standard deviation $\left(\sigma_{\mu C T}\right)$ values of the $\mu \mathrm{CT}$ segmentation volume. The thresholds were: 1) $T_{\mu C T}$, 2) $T_{\mu C T}-\sigma_{\mu C T}$, and 3) $T_{\mu C T}-2 \sigma_{\mu C T}$. Surface generation and intensity measurements were performed in the same manner as was carried out on the phantom images. The apparent (radiological) mass was calculated as described for the $\mu \mathrm{CT}$ reference segmentations. The thickness error was evaluated as the "surface distance" between that of the $\mu \mathrm{CT}$ generated volumetric surfaces and those of the $\mathrm{CT}_{0}$ and $\mathrm{CT}_{\text {deblurred }}$ volumes. This measurement was taken to be the mean of all the shortest 3-D lines from each vertex of the $\mu \mathrm{CT}$ to the closest vertex found on the $\mathrm{CT}_{0}$ and $\mathrm{CT}_{\text {deblurred }}$ surfaces ( $n>300,000$ ). The thickness error is included in addition to the mass error because it is highly sensitive to the 3-D geometrical contour of the cortical bones by virtue of the accurate registrations of the $\mu \mathrm{CT}$, while the volume measurement from which the mass value is derived is insensitive to the geometric outline. Agreement between CT and $\mu \mathrm{CT}$ image intensities was measured using a linear regression fit between the intensity values of all voxels within the $\mu \mathrm{CT}$ cortical segmentation versus spatially-mutual voxel intensity values in the $\mathrm{CT}_{0}$ and $\mathrm{CT}_{\text {deblurred }}$ volumes.

An ANOVA model (that included the threshold used to define cortical bone and a factor indicating deblurred/original) was used for each quality metric: surface distance error, 
radiological mass error and voxel intensity correlation to examine differences in the cadaver experiment (JMP, SAS, Cary, NC, USA). A separate ANOVA analysis was conducted for each of the five bone regions.

\section{Results}

\section{Phantom experiment}

Deblurring improved the accuracy of both thickness and intensity measures at the expense of increased cortical bone intensity noise for all algorithms (Thickness error $=0.16-0.37 \mathrm{~mm}$, $\mathrm{p}<0.001$, Intensity error $=152-247 \mathrm{HU}, \mathrm{p}<0.001)$, (Table 3). For a number of the images, especially at the initial iterations, no segmentation could be created at even the minimum threshold value due to the diffused and suppressed intensity values of the thin layers. In these cases, the errors were reported to be $100 \%$, and the intensity variance was set to be that of the entire image. In other cases, with two simulated cortical layers, the diffused intensity of the bilayers resulted in their being lumped together as one layer. The intensity and thickness of these constructs were reported as is by the automatic segmentation. The estimated Gaussian PSF's for the cortical phantom images had a FWHM equal to $1.13 \pm 0.07$ and $1.11 \pm 0.08 \mathrm{~mm}$ within the transverse and coronal/sagittal planes, respectively.

The Blind algorithms produced the noisiest results. The RL and RL-TV1 groups had nearly identical performance measures in all 3 performance measures. Adding regularization to the RL algorithm did not result in improved accuracy of thickness or intensity nor did it meaningfully decrease intensity standard deviation. The MRNSD, CGLS, and RL-TV3 algorithms had minimal increases in their intensity standard deviation combined with improvements in thickness error and intensity error compared to the original images, an indication that there was a net gain in restoration of details, with a negligible increase in noise or partial volume artefacts. These three methods also produced very similar error reductions in intensity values. MRNSD and RL performed similarly in thickness and intensity error reduction. Overall, the MRNSD method had the best combination of performance metrics (Table 3), having an edge over RL in intensity standard deviation.

On the phantom images, every 50 iterations of deconvolution required approximately 30 minutes of computation time on the workstation, and this time increased linearly with additional 
iterations. The larger phantom images required substantially longer times as the matrix multiplication operations saturated the available $32 \mathrm{GBs}$ of RAM. Those algorithms which were implemented in ImageJ iterated more rapidly, but the processes were also quicker to overwhelm the available memory compared to those implemented in MATLAB. Overall, no clear benefit was evident regarding the relative computational economy of the different methods.

\section{Cadaveric CMFS experiment}

MRNSD deconvolution improved the accuracy of thickness and intensity measurements as well as the topology of segmentations obtained from the cadaveric CT volumes (Figures 5 and 6, Table 4). Deconvolution restored much of the thin cortical architecture. Significant $(p<0.001)$ recoveries in the geometrical and intensity-derived density values were observed for all measures (Table3). ANOVA showed that both deblurring and the threshold used had significant effects on surface distance and radiological mass errors as well as on voxel intensity correlation in all regions. In the clinical CT image of the cadaver, the PSF FWHM was $1.32 \pm 0.06 \mathrm{~mm}$ in the transverse planes, and $1.27 \pm 0.1 \mathrm{~mm}$ longitudinally.

The overall mean cortical thickness error was reduced by $3.8 \pm 1.5 \mathrm{~mm}$ by the deconvolution process (Table 3). The mean thickness of the $\mu \mathrm{CT}$ reference volumes was $1.08 \pm 0.77 \mathrm{~mm}$. The error in radiological mass measurement was reduced by $0.14 \pm 0.05 \mathrm{~g}$ in the deblurred images, with the $\mu \mathrm{CT}$ truth reference having a radiological mass of $0.37 \pm 0.21 \mathrm{~g}$. The voxel intensity correlation in the deblurred CT images was improved to $r=0.2 \pm 0.14$ from $0.09 \pm 0.16$ in the original images.

\section{Discussion}

As a whole, the deconvolution methods using PSF estimation employed in this study produced a marked and measurable degree of restoration in the phantoms and CMFS anatomical data which was inaccessible in the original, non-deblurred images (Figures 3,5 and 6). The comprehensive series of objective metrics enabled direct comparisons quantifying the accuracy of thin cortical bone geometry and density measurements. 
This study was not an exhaustive exploration of all deconvolution algorithms, however, this analysis has elucidated trade-offs between the algorithms. Specifically this analysis highlights the need to be cautious when employing approaches that might appear to offer cosmetic enhancement, while altering the information originally available in the image to an unknown degree ${ }^{44}$.

The phantom study revealed significant differences in performance between deconvolution methods even between methods that relied on a measured PSF. Rigorous mathematical deduction for predicting performance of iterative algorithms for a specific scenario is non-trivial ${ }^{42}$ and likely problem specific. RL, MRNSD and CGLS all showed significant and meaningful improvements in intensity and thickness estimation in clinical CT of the CMFS. RL (with no regularization) and MRNSD both had low intensity and thickness errors, while MRNSD also had the benefit of reduced intensity variation at the cost of qualitatively more ringing artifact. CGLS was also useful at improving thickness measurements but had greater error in intensity restoration.

The differences observed between the different regularization parameters $(\lambda)$ within Richardson-Lucy with Total Variation regularization suggest that there was little gain from total variation regularization in improving intensity or thickness estimates when restoring thin cortical bones. As expected total variation regularization did decrease intensity standard deviation because it imposes a penalty on intensity variance, thereby smoothing.

The Blind deconvolution method used an iterative maximum likelihood approach to recover images, as well as the PSF ${ }^{18}$. Multiple studies have applied deconvolution for deblurring of mineralization and general restoration of clinical CT resolution using Blind PSF-based methods 15,33. The thickness measurements with the Blind method appear to be competitive with the CGLS and MRNSD algorithms (Table 3), however this improvement in geometrical information comes at the cost of highly exaggerated edge enhancement, with great distortion of the HU values beyond physically interpretable levels (as measured by the variance values in Table 3). Furthermore, the Blind algorithm's results were highly unstable, iterating towards exponentially increasing intensity errors.

This investigation calculated PSF from specific features within images that allow convenient estimates. The most accurate method of obtaining the PSF is by direct measurement using a slitphantom. However, such a process is non-trivial and complicated by its 3 -dimensionality ${ }^{5}$. Image PSFs are also affected by subject and scanner parameters (e.g. filter type and beam energy). In this study, the PSF is obtained from the same image that is analyzed without any 
scanner specific measurement beyond the associated metadata, but is limited by simplifications assumed about the 3D characteristics of the $\mathrm{PSF}^{22,28,35}$.

Applying different reconstruction kernels during filtered back projection (FBP) is distinct to image restoration by deconvolution. Changes in reconstruction kernels (bone, sharp, soft-tissue) applied during FBP are applied in the projection space on raw data from the scanner and also result in a trade-off between noise and spatial resolution. Within this investigation the standard kernel was used in the iterative deconvolution methods. The standard kernel was chosen for this investigation because of previous work within this group has shown that PSF estimation was more accurate when using the standard vs bone kernel reconsructions ${ }^{28}$ ( this was likely due to the non-gaussian PSF shape of the bone kernel reconstructed volumes). Further the benefits of iterative deconvolution are not well matched to the bone kernel reconstructions. Bone kernel scans are already noisy and deconvolution tends to increase noise, although geometric restoration may be possible, the scans are already sharp, and therefore there will likely be less benefit. This investigation was focused on quantitatively restoring both intensity and geometry by iterative deconvolution. Bone kernels reconstructions are less well matched to quantitative intensity reconstruction, and instead bone kernel reconstruction is focused on better visualization of fine structure. Bone kernel reconstructions are known to cause intensity bias at edges.

Clinically, the kernel choice is driven by the diagnostic protocol, such as using sharp kernels for detecting bone and high-contrast nodules, or a more averaging-type kernel for better tissuelevel contrast. Ohkubo et al showed that different kernel reconstructions can be reproduced with reasonable accuracy from one another if reasonably precise measures of the PSF are available ${ }^{26}$. Combination of these filters in a weighted manner might also provide a perceived improvement in image quality ${ }^{46}$. However, iterative deconvolution can improve upon the results of linear FBP kernels as it allows for non-linear trade-offs of noise and sharpness that can retrieve a more "true" image of an object. Indeed this is consistent with findings on the trade-offs between standard and sharper (bone) kernels that can double resolution at the expense of quadrupling noise in intensity ${ }^{39}$. In this investigation we showed an increase of 2-3times in the accuracy of thickness measurements with improved estimation of intensity and increased noise. An enhanced 3D PSF estimation algorithm that better represents the physics of the X-ray CT image 
acquisition process and development of an iterative inverse-solver tailored to the noise statistics of CT images may further improve the iterative deconvolution.

Iterative reconstruction (IR) algorithms such as adaptive statistical iterative reconstruction (ASIR) and model based iterative reconstruction (MBIR) are being employed in newer CT scanners to improve image quality ${ }^{8,9,13,14,36}$. In these methods the FBP image is first created and used as the starting point for the subsequent iterations. The objective of both IR and iterative deconvolution is to find an optimal estimate of the true object $f_{\text {true }}$ sampled and blurred by $K$ in presence of noise $\varepsilon$. IR algorithms have the advantage of direct access to raw pre-reconstruction projection data and a sophisticated account of system properties, whereas the iterative deconvolution approach used in this study employ only a post-sampled FBP image to estimate the PSF and a generic noise distribution model. Therefore IR methods theoretically can produce superior quantification of bone intensity and shape, at the expense of increased complexity, computational time and with more scanner specific elements. The advantage of the methodology presented in this manuscript is the breadth of potential use, many images can be restored without direct knowledge of the underlying scanning system or raw-imaging data as well as the wide availability of deconvolution methods. Further IR generated volumes may be improved by further deconvolution with appropriately estimated PSFs.

The accuracy of the CMFS thin bone cortical geometries in the volumetric secondary constructions of the deblurred images by means of automatic segmentation was improved by $81 \%$ in the deblurred images and the total bone mineral mass recovery was $32 \%$. Some regions of the maxillary sinus were $<0.25 \mathrm{~mm}$ thick (1/4 of the slice thickness) and its surface was oriented parallel to the transverse planes. Such loss of information due to severe under-sampling cannot be recovered by deconvolution, as the intensity values have fallen below the noise level in the surrounding medium. This explains the ubiquitous appearance of false foramens on anterior maxillary sinus walls, orbital floors, and other thin cortical regions in clinical CT of the $\mathrm{CMFS}^{6,25,38,45}$.

Iterative deconvolution methods, using estimated PSFs significantly restored geometrical and radiological density information both quantitatively and qualitatively in thin osseous features. The MRNSD algorithm resulted in the highest relative deblurring performance as assessed by a cortical bone thickness error and intensity error. Error was assessed against known values in a cortical bone mimicking phantom and against $\mu \mathrm{CT}$ 
images of excised thin cortical bone samples from the skull. Using iterative deconvolution methods resulted in an $82 \%$ reduction in cortical thickness error and 55\% reduction in bone mineral mass measurement. The methodology outlined in this investigation demonstrates a practical and powerful method for quantitatively restoring CT information that may be useful for diagnosis, treatment planning and finite element modeling.

\section{Acknowledgements}

This study was funded by the Natural Science and Engineering Research Council of Canada and the Ontario Graduate Scholarship.

\section{References}

1. Baino, F. Biomaterials and implants for orbital floor repair. Acta Biomater. 7:3248-3266, 2011.

2. Bardsley, J. M. Applications of a nonnegatively constrained iterative method with statistically based stopping rules to CT, PET, and SPECT imaging. Electron. Trans. Numer. Anal. 38:34-43, 2011.

3. Bardsley, J. M., and J. G. Nagy. Covariance-preconditioned iterative methods for nonnegatively constrained astronomical imaging. SIAM J. matrix Anal. Appl. 27:11841197, 2006.

4. Berisha, S., and J. Nagy. Iterative methods for image restoration. ... Signal Process. Vol. $4 \quad$ Image, $\quad \ldots \quad 1-59, \quad$ 2013.at <http://books.google.com/books?hl=en\&lr=\&id=QJ3HqmLG8gIC\&oi=fnd\&pg=PA193 $\&$ dq=Iterative+Methods+for+Image+Restoration\&ots=RHpzfDVSi1 \&sig=srDAMuvY RwHsdCQ4_Gq7tfCFK_Q>

5. Boone, J. M. Determination of the presampled MTF in computed tomography. Med. Phys. 28:356-360, 2001.

6. Chang, P. S.-H., T. H. Parker, C. W. Patrick, and M. J. Miller. The accuracy of stereolithography in planning craniofacial bone replacement. J. Craniofac. Surg. 14:164170, 2003.

7. Erlandsson, K., I. Buvat, P. H. Pretorius, B. a Thomas, and B. F. Hutton. A review of partial volume correction techniques for emission tomography and their applications in neurology, cardiology and oncology. Phys. Med. Biol. 57:R119-59, 2012.

8. Geleijns, J., and R. Irwan. Practical Approaches to Dose Reduction: Toshiba Perspective. In: Radiation Dose from Multidetector CT. Berlin: Springer, 2012.doi:10.1007/174

9. Gervaise, A., B. Osemont, S. Lecocq, A. Noel, E. Micard, J. Felblinger, and A. Blum. CT image quality improvement using Adaptive Iterative Dose Reduction with widevolume acquisition on 320-detector CT. Eur. Radiol. 22:295-301, 2012. 
10. Hansen, P. C., J. G. Nagy, and D. O’Leary. Deblurring Images: Matrices, Spectra, and Filtering. Fundamentals of Algorithms 3, SIAM, Philadelphia, 2006, 2006.

11. Helgason, B., F. Taddei, E. Schileo, L. Cristofolini, M. Viceconti, H. Pálsson, and S. Brynjólfsson. A modified method for assigning material properties to FE models of bones. Med. Eng. Phys. 30:444-453, 2008.

12. Hsieh, J. Computed Tomography, Second Edition. Bellingham, WA: SPIE, 2009.doi:10.1117/3.817303

13. Hsieh, J., B. Nett, Z. Yu, K. Sauer, J.-B. Thibault, and C. a. Bouman. Recent Advances in CT Image Reconstruction. Curr. Radiol. Rep. 1:39-51, 2013.

14. Irwan, B., S. Nakanishi, and A. Blum. AIDR 3D-Reduces Dose and Simultaneously Improves Image Quality. Toshiba Med. Syst. Whitepaper 1-8, 2012.at $<$ http://scholar.google.com/scholar?hl=en\&btnG=Search\&q=intitle:AIDR+3D++Reduces+Dose+and+Simultaneously+Improves+Image+Quality\#0>

15. Jiang, M., G. Wang, M. W. Skinner, J. T. Rubinstein, and M. W. Vannier. Blind deblurring of spiral CT images. IEEE Trans. Med. Imaging 22:837-845, 2003.

16. Kawata, Y., Y. Nakaya, N. Niki, H. Ohmatsu, K. Eguchi, M. Kaneko, and N. Moriyama. Measurement of three-dimensional point spread functions in multidetector-row CT. , 2008.

17. Kobayashi, F., O. Sasaki, S. Nakajima, and J. Ito. Measurement of layer thickness using spread width of longitudinal image in helical CT. Oral Radiol. 15:85-93, 1999.

18. Lagendijk, R. L., J. Biemond, and D. E. Boekee. Identification and restoration of noisy blurred images using the expectation-maximization algorithm. IEEE Trans. Acoust. 38:1180-1191, 1990.

19. Lohfeld, S., V. Barron, and P. E. McHugh. Biomodels of bone: a review. Ann. Biomed. Eng. 33:1295-1311, 2005.

20. Maloul, A., J. Fialkov, and C. Whyne. The impact of voxel size-based inaccuracies on the mechanical behavior of thin bone structures. Ann. Biomed. Eng. 39:1092-1100, 2011.

21. Meijering, E. H. W., W. J. Niessen, J. P. W. Pluim, and M. A. Viergever. Quantitative comparison of sinc-approximating kernels for medical image interpolation., 1999.

22. Meinel, J. F., G. Wang, M. Jiang, T. Frei, M. Vannier, and E. Hoffman. Spatial variation of resolution and noise in multi-detector row spiral CT. Acad. Radiol. 10:607-613, 2003.

23. Mustafa, S. F., P. L. Evans, A. Bocca, D. W. Patton, A. W. Sugar, and P. W. Baxter. Customized titanium reconstruction of post-traumatic orbital wall defects: a review of 22 cases. Int. J. Oral Maxillofac. Surg. 40:1357-1362, 2011.

24. Newman, D. L., G. Dougherty, A. al Obaid, and H. al Hajrasy. Limitations of clinical CT in assessing cortical thickness and density. Phys. Med. Biol. 43:619-626, 1998.

25. Nunery, W. R., P. J. Timoney, and H. B. H. Lee. General Principles of Management of Orbital Fractures. In: Smith and Nesi's Ophthalmic Plastic and Reconstructive Surgery, edited by E. H. Black, F. A. Nesi, G. J. Gladstone, and M. R. Levine. New York, NY: Springer New York, 2012.doi:10.1007/978-1-4614-0971-7

26. Ohkubo, M., S. Wada, A. Kayugawa, T. Matsumoto, and K. Murao. Image filtering as 
an alternative to the application of a different reconstruction kernel in CT imaging: Feasibility study in lung cancer screening. Med. Phys. 38:3915, 2011.

27. Pakdel, A., J. G. Mainprize, N. Robert, J. Fialkov, and C. M. Whyne. Model-based PSF and MTF estimation and validation from skeletal clinical CT images. Med. Phys. 41:011906, 2014.

28. Pakdel, A., J. G. Mainprize, N. Robert, J. Fialkov, and C. M. Whyne. Model-based PSF and MTF estimation and validation from skeletal clinical CT images. Med. Phys. 41:11906, 2014.

29. Pakdel, A., N. Robert, J. Fialkov, A. Maloul, and C. Whyne. Generalized method for computation of true thickness and x-ray intensity information in highly blurred submillimeter bone features in clinical CT images. Phys. Med. Biol. 57:8099-116, 2012.

30. Papadopoulos, M. a., P. K. Christou, P. K. Christou, A. E. Athanasiou, P. Boettcher, H. F. Zeilhofer, R. Sader, and N. a. Papadopulos. Three-dimensional craniofacial reconstruction imaging. Oral Surgery, Oral Med. Oral Pathol. Oral Radiol. Endodontology 93:382-393, 2002.

31. Pinheiro, M., and J. L. Alves. A new Level-set based Protocol for Accurate Bone Segmentation from CT Imaging. ArXiv e-prints , 2015.

32. Prevrhal, S., K. Engelke, and W. A. Kalender. Accuracy limits for the determination of cortical width and density: the influence of object size and CT imaging parameters. Phys. Med. Biol. 44:751-764, 1999.

33. Rollano-Hijarrubia, E., R. Manniesing, and W. J. Niessen. Selective deblurring for improved calcification visualization and quantification in carotid CT angiography: validation using micro-CT. IEEE Trans. Med. Imaging 28:446-453, 2009.

34. Schneider, C. A., W. S. Rasband, and K. W. Eliceiri. NIH Image to ImageJ: 25 years of image analysis. Nat. Methods 9:671-675, 2012.

35. Schwarzband, G., and N. Kiryati. The point spread function of spiral CT. Phys. Med. Biol. 50:5307-5322, 2005.

36. Silva, A. C., H. J. Lawder, A. Hara, J. Kujak, and W. Pavlicek. Innovations in CT dose reduction strategy: application of the adaptive statistical iterative reconstruction algorithm. AJR. Am. J. Roentgenol. 194:191-199, 2010.

37. Starck, J. L., E. Pantin, and F. Murtagh. Deconvolution in Astronomy: A Review. Publ. Astron. Soc. Pacific 114:1051-1069, 2002.

38. Szwedowski, T. D. T. D. Development and validation of a subject-specific finite element model of the human craniofacial skeleton. , 2007.at <http://www.csa.com/partners/viewrecord.php?requester=gs\&amp;collection=TRD\&a mp;recid=9113853MT>

39. Thibault, J.-B., K. D. Sauer, C. a. Bouman, and J. Hsieh. A three-dimensional statistical approach to improved image quality for multislice helical CT. Med. Phys. 34:4526, 2007.

40. Treece, G. M., a. H. Gee, P. M. Mayhew, and K. E. S. Poole. High resolution cortical bone thickness measurement from clinical CT data. Med. Image Anal. 14:276-290, 2010.

41. Varghese, B., D. Short, R. Penmetsa, T. Goswami, and T. Hangartner. Computed- 
tomography-based finite-element models of long bones can accurately capture strain response to bending and torsion. J. Biomech. 44:1374-1379, 2011.

42. Vio, R., J. Bardsley, and W. Wamsteker. Least-squares methods with Poissonian noise: Analysis and comparison with the Richardson-Lucy algorithm. Astron. Astrophys. 436:741-755, 2005.

43. Vogel, C. R. Computational Methods for Inverse Problems. SIAM, 2002.

44. Wang, G., M. W. Vannier, M. W. Skinner, M. G. P. Cavalcanti, and G. W. Harding. Spiral CT image deblurring for cochlear implantation. IEEE Trans. Med. Imaging 17:251-262, 1998.

45. Westin, C. F., A. Bhalerao, H. Knutsson, and R. Kikinis. Using local 3D structure for segmentation of bone from computer tomography images. , 1997.doi:10.1109/CVPR.1997.609418

46. Wildberger, J. E., A. H. Mahnken, T. Flohr, R. Raupach, C. Weiss, R. W. Günther, and S. Schaller. Spatial domain image filtering in computed tomography: feasibility study in pulmonary embolism. Eur. Radiol. 13:717-723, 2003.

47. Zannoni, C., R. Mantovani, and M. Viceconti. Material properties assignment to finite element models of bone structures: a new method. Med. Eng. Phys. 20:735-740, 1998. 

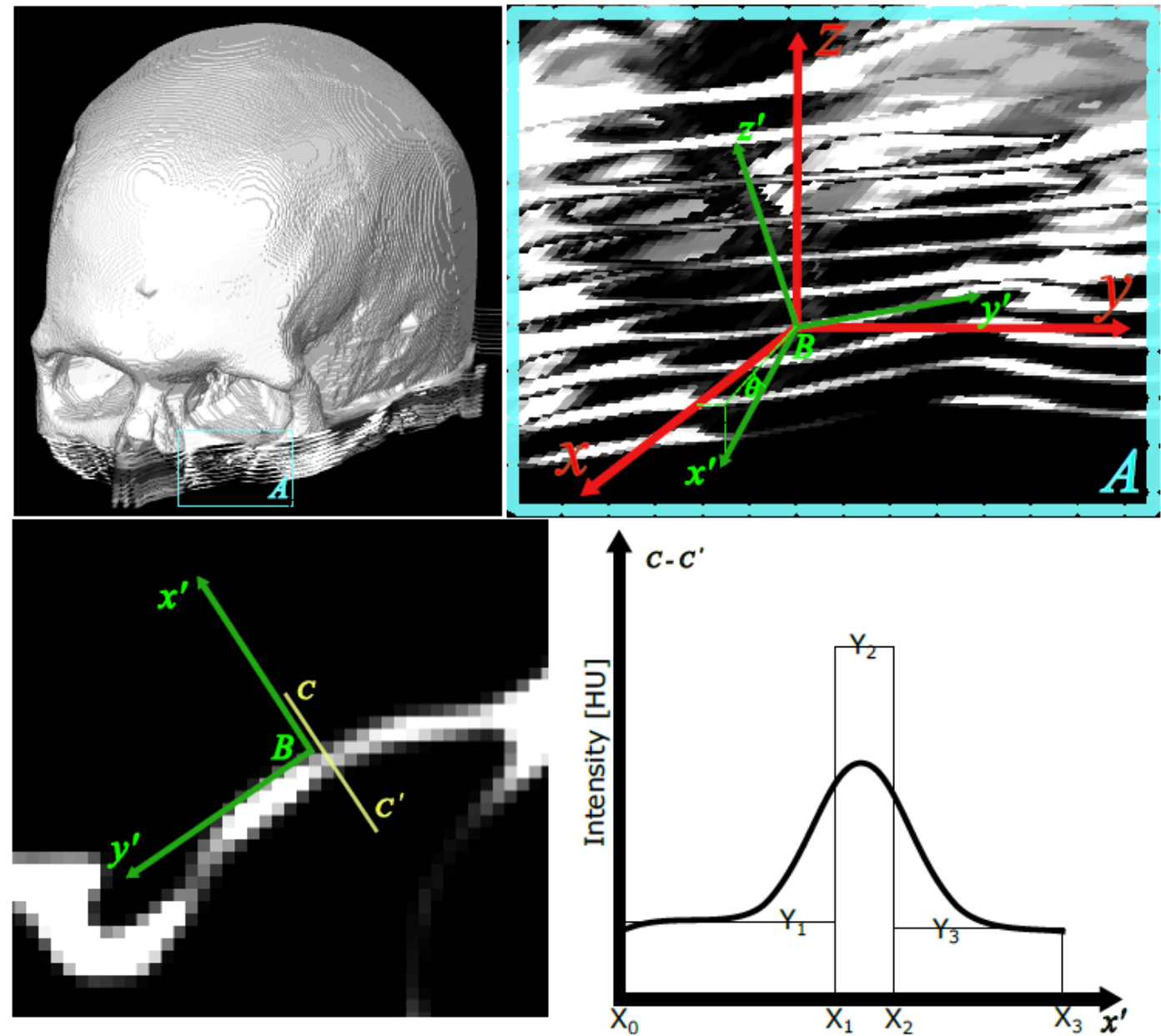

Figure 1: Illustration of the intensity profile extraction process. The anatomical regions which might be good candidates are known a priori, such as the thin anterior maxillary sinus wall (Box A, top left), as shown on the $3 \mathrm{D}$ reconstruction of the superior portion of the cadaveric CFS. The image axes are denoted as $x, y$, and $z$, while $x^{\prime}, y^{\prime}$ and,$z^{\prime}$ are the rotated axes at point $\mathrm{B}$, forming angle $\theta$ with respect to the transverse slices (top right). An interpolated slice through the voxels illustrates the line (c->c') used for profile extraction in the plane of the $x^{\prime}$ and $y^{\prime}$ (bottom left). The intensity plot of the extracted profile, along with the characteristic variables used for the PSF estimation (bottom right). 
Teflon (cortical bone)

Delrin (trabecular bone)

Water equivalent plastic (tissue)
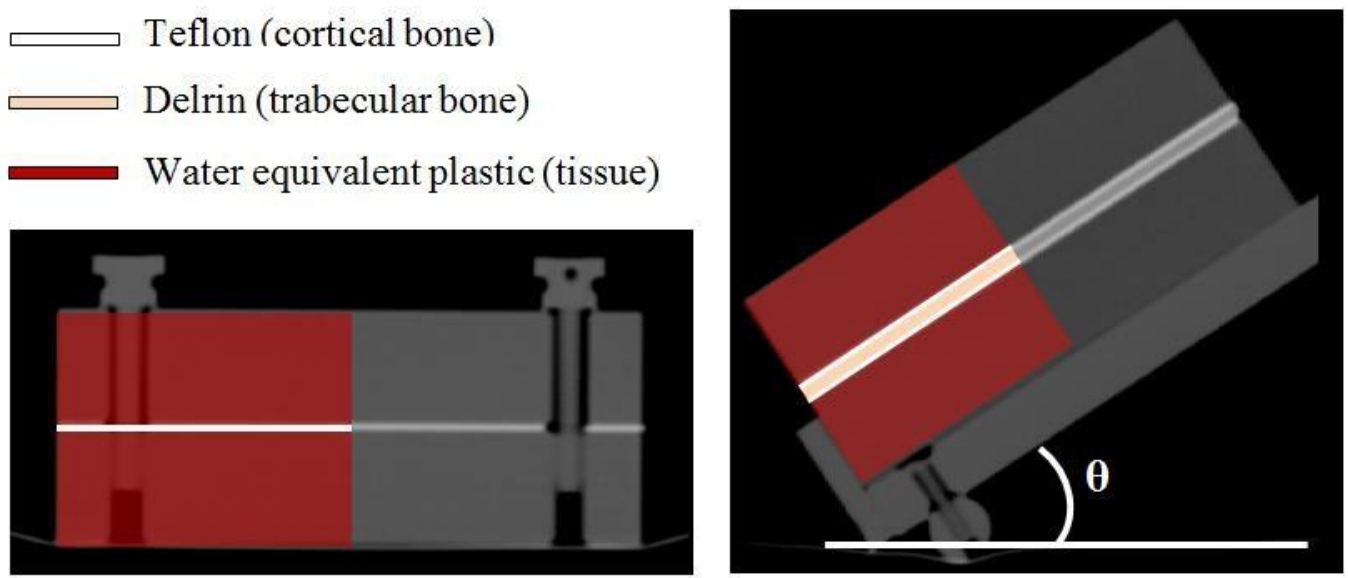

Figure 2: Sagittal slice views from two of the sample phantoms, with a color legend overlay highlighting the material layers. The left and right are tilted $\theta=0^{\circ}$ and $\theta=35^{\circ}$ degrees away from the coronal plane. 


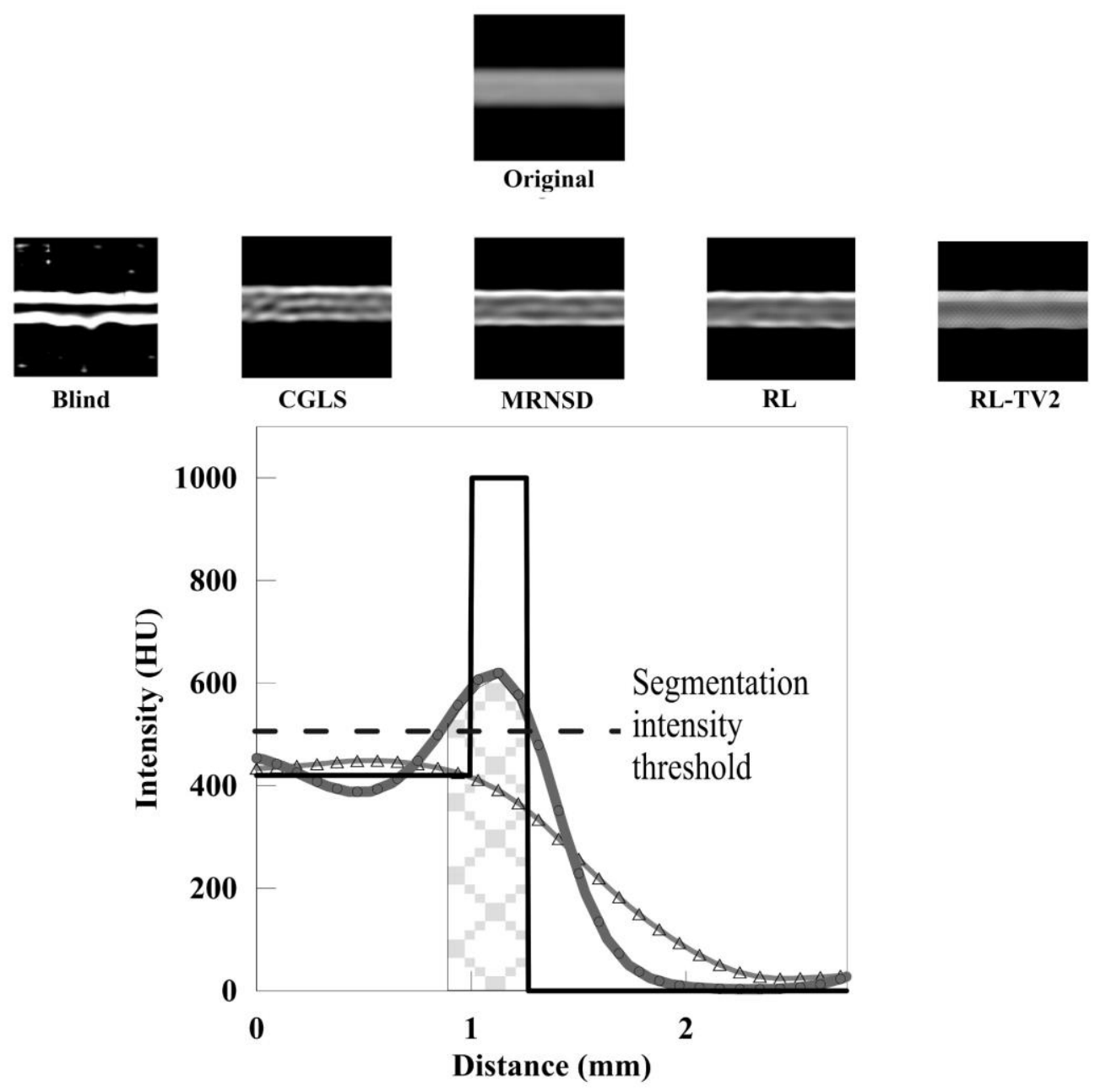

Figure 3: Top: sample results for different deconvolution algorithms of phantom 1 after 100 iterations. Bottom: an intensity profile sampled from one cortical edge to the middle of the phantom, taken from the original image (triangle symbols), and the same image deblurred by 100 iterations of CGLS deconvolution (grey line). The rectangular plot represents the true intensity profile. The vertical edges define the thickness, and the height is the measured (theoretical) intensity of Teflon. The hashed region represents the thickness of the deblurred volume, whose edges are defined by the intersection of corresponding profile at the given intensity threshold. The mean intensity and the variance of the deblurred data set is calculated from the mean and variance within this region. 


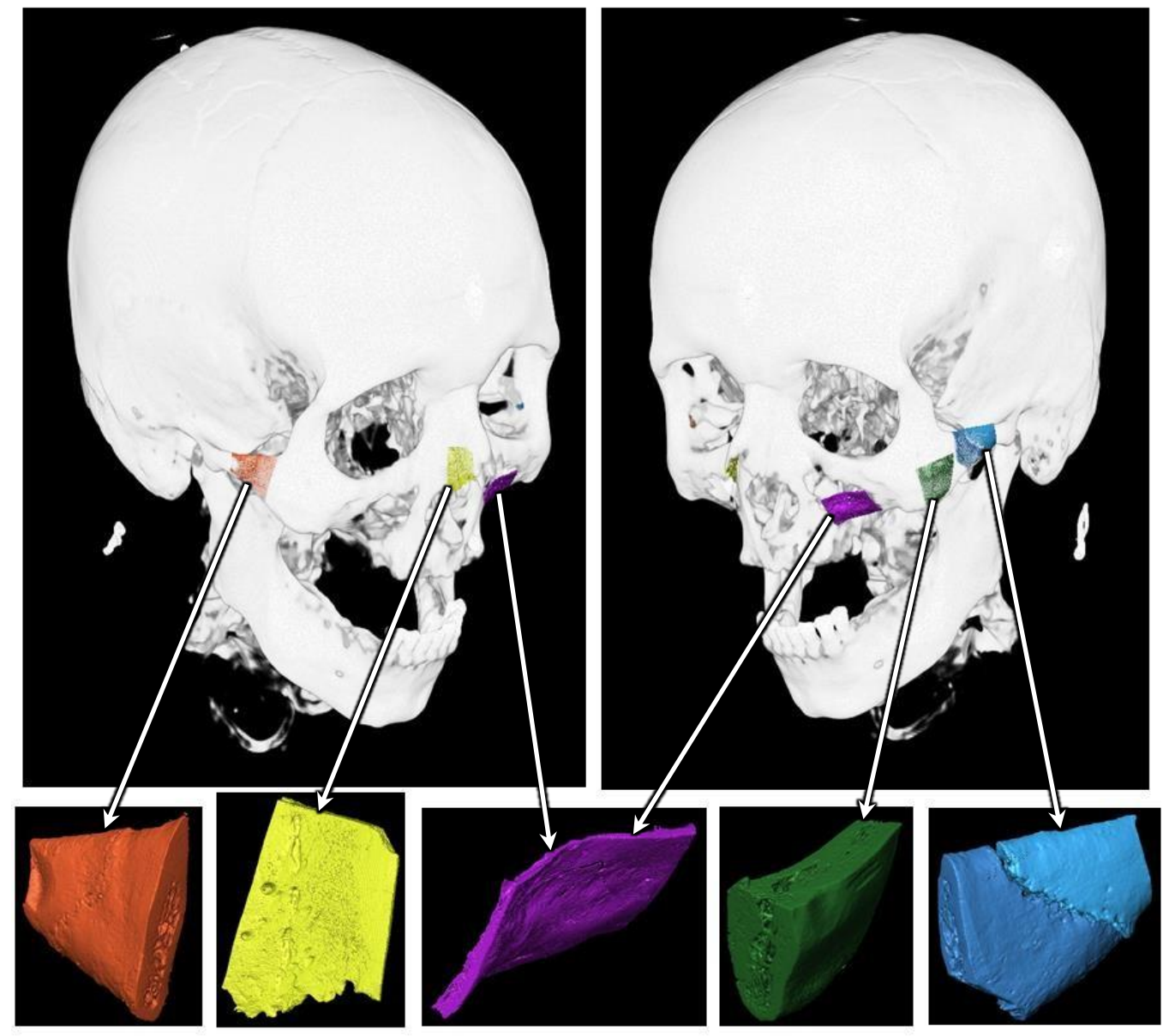

Figure 4: Surface rendering of the cadaveric specimen clinical CT with the $\mu$ CT volumes of the excised cortical samples registered to their origins. The cortical samples are from the right temporal process of the zygomatic bone (orange), the nasal bone bordering on the frontal process of the maxilla (yellow), the frontal maxillary sinus wall (purple), the left lateral process of the zygomatic bone (green), and the left portion of the zygomatic arch containing the zygomaticotemporal suture. 

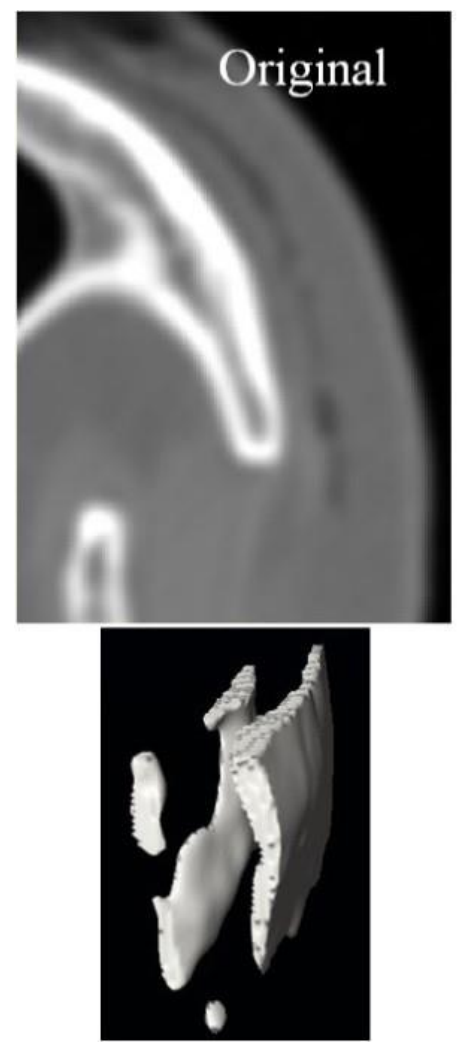
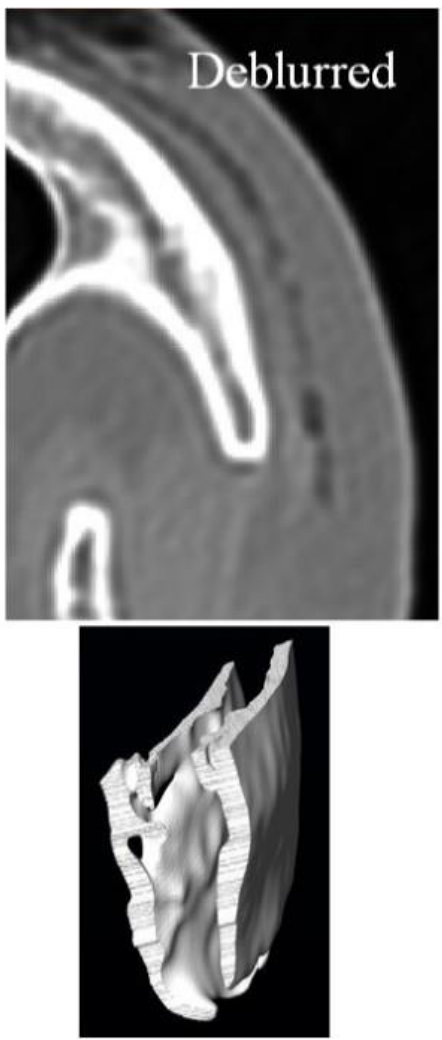
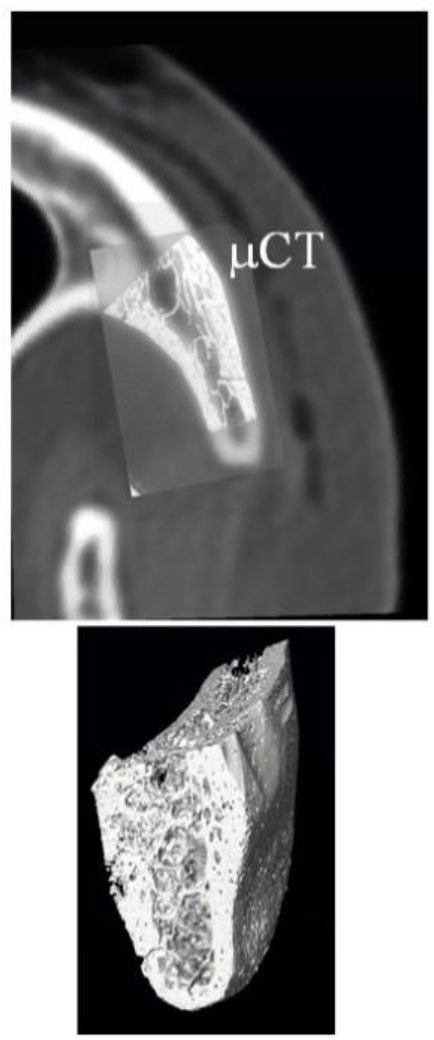

Figure 5: Transverse slice from the zygomatic bone in the original CT (top left), the deblurred results (top middle), and the registered $\mu \mathrm{CT}$ image slice after resampling into the common coordinate system (top right). The bottom row demonstrates the associated segmented cortical volumes at a threshold value of $1200 \mathrm{HU}$. 

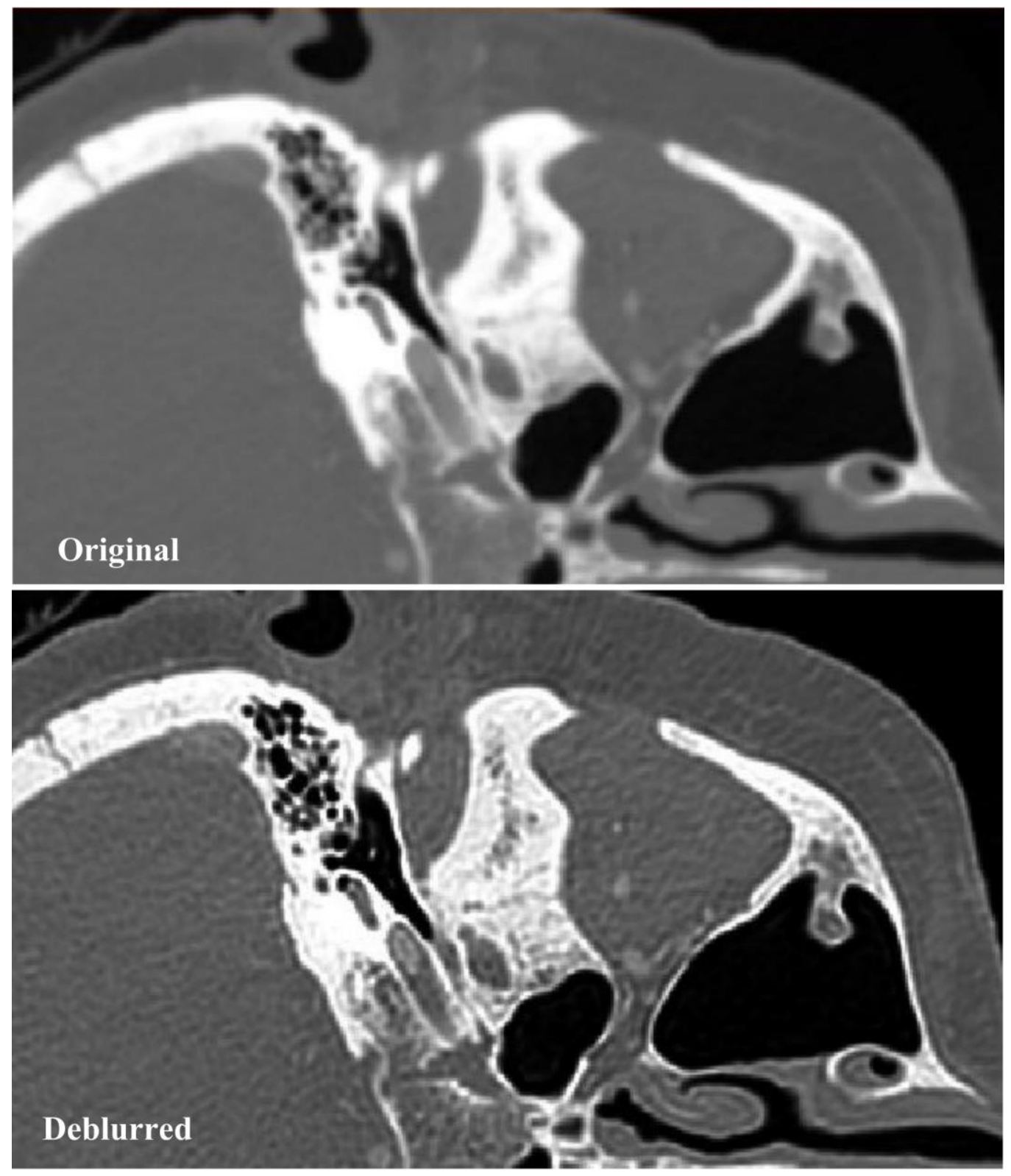

Figure 6: Comparison of an original CT slice with the deconvolution result (100 iterations, MRNSD). 
Table 1: Deconvolution algorithms for deblurring of phantom images

\begin{tabular}{lll}
\hline Algorithm & Implementation & Reference \\
\hline Blind & MATLAB & (Fish et al., 1995) \\
RL-TV4 $(\lambda=0.1)$ & ImageJ-Deconvolution Lab & (Schmit, 2007), (Dey et al., 2006) \\
RL-TV3 $(\lambda=0.01)$ & ImageJ-Deconvolution Lab & (Schmit, 2007), (Dey et al., 2006) \\
RL-TV2 $(\lambda=0.001)$ & ImageJ-Deconvolution Lab & (Schmit, 2007), (Dey et al., 2006) \\
RL-TV1 $(\lambda=0.0001)$ & ImageJ-Deconvolution Lab & (Schmit, 2007), (Dey et al., 2006) \\
RL & MATLAB & (Richardson, 1972; Lucy, 1974) \\
MRNSD & MATLAB-RestoreTools & (Berisha and Nagy, 2013) \\
CGLS & MATLAB-RestoreTools & (Berisha and Nagy, 2013) \\
\hline
\end{tabular}

Table 2: Configuration of simulated shell bone layers in phantoms. The angle $\theta$ determines the phantom tilt as illustrated Figure 3.

\begin{tabular}{ccc}
\hline Phantom & $\begin{array}{c}\text { Cortical/Trabecular/Cortical } \\
\text { Thickness (mm) }\end{array}$ & $\theta\left(^{\circ}\right)$ \\
\hline 1 & $0.25 / 2.36 / 0.25$ & 0 \\
2 & $0.50 / 1.02 / 0.50$ & 0 \\
3 & $0.79 / 0.00 / 0.00$ & 0 \\
4 & $0.25 / 0.00 / 0.00$ & 35 \\
5 & $0.50 / 1.02 / 0.50$ & 35 \\
6 & $0.79 / 2.36 / 0.79$ & 35 \\
\hline
\end{tabular}

Table 3: Performance metrics for each algorithm at restoring images of phantom data. abcdeRepresent groups of algorithms that performance significantly different based on pairwise t-tests $(\mathrm{p}<0.001)$.

\begin{tabular}{|c|c|c|c|c|c|c|c|}
\hline \multirow[t]{2}{*}{$\begin{array}{l}\text { Test } \\
\text { group }\end{array}$} & \multirow[t]{2}{*}{$\begin{array}{l}\text { Iterations to } \\
\text { Convergence }\end{array}$} & \multicolumn{2}{|c|}{$\begin{array}{l}\text { Thickness error } \\
\text { (mm) }\end{array}$} & \multicolumn{2}{|c|}{ Intensity error (HU) } & \multicolumn{2}{|c|}{$\begin{array}{c}\text { Intensity STD } \\
(\mathrm{HU})\end{array}$} \\
\hline & & Mean & SD & Mean & SD & Mean & SD \\
\hline Original & NA & $0.45^{\mathrm{a}}$ & 0.41 & $853^{a}$ & 803 & $19^{a}$ & 17 \\
\hline Blind & 82 & 0.19 bde & 0.16 & $225^{b}$ & 181 & $451^{\mathrm{b}}$ & 438 \\
\hline RL-TV4 & 25 & $0.37^{\mathrm{c}}$ & 0.33 & $174^{\text {bd }}$ & 133 & $378^{c}$ & 363 \\
\hline RL-TV3 & 113 & $0.22^{\text {bd }}$ & 0.19 & $230^{\text {bd }}$ & 185 & $148^{\mathrm{ae}}$ & 98 \\
\hline RL-TV2 & 126 & $0.21^{\mathrm{d}}$ & 0.18 & $230^{\mathrm{bc}}$ & 183 & $247^{d}$ & 241 \\
\hline RL-TV1 & 94 & $0.22^{\mathrm{bd}}$ & 0.19 & $215^{\text {bd }}$ & 160 & $252^{\mathrm{d}}$ & 226 \\
\hline RL & 78 & $0.21^{\text {bd }}$ & 0.17 & $177^{\mathrm{d}}$ & 132 & $234^{d}$ & 207 \\
\hline MRNSD & 100 & $0.18^{\text {be }}$ & 0.15 & $152^{\mathrm{d}}$ & 109 & $108^{\mathrm{ae}}$ & 4 \\
\hline CGLS & 125 & $0.16^{\mathrm{e}}$ & 0.12 & $247^{c}$ & 201 & $174^{\mathrm{e}}$ & 133 \\
\hline
\end{tabular}


Table 4: Summary from the cadaveric CMFS specimens, compared to corresponding non-deblurred CT image. The ANOVA models showed significant differences in least square (LSQ) means in Surface distance error, Radiological mass error and voxel intensity correlation $(\mathrm{P}<<0.001)$ between the original and deblurred scans.

\begin{tabular}{|c|c|c|c|c|c|c|c|c|c|c|}
\hline \multirow{3}{*}{ Region } & \multirow{2}{*}{\multicolumn{2}{|c|}{$\begin{array}{l}\text { Summary Statistics } \\
\text { uCT Thickness }(\mathrm{mm})\end{array}$}} & \multirow{3}{*}{$\begin{array}{l}\text { Radiological } \\
\text { Mass (g) }\end{array}$} & & \multicolumn{6}{|c|}{ ANOVA Results } \\
\hline & & & & \multicolumn{3}{|c|}{$\begin{array}{l}\text { Surface distance } \\
\text { error }(\mathrm{mm})\end{array}$} & \multicolumn{2}{|c|}{$\begin{array}{c}\text { Radiological } \\
\text { mass error }(\mathrm{mm})\end{array}$} & \multicolumn{2}{|c|}{$\begin{array}{l}\text { Voxel intensity } \\
\text { correlation }\end{array}$} \\
\hline & Mean & SD & & Group & LSQ mean & Std. err. & LSQ mean & Std. err. & LSQ mean & Std. error \\
\hline \multirow{2}{*}{ Nasal } & \multirow{2}{*}{1.30} & \multirow{2}{*}{0.68} & \multirow{2}{*}{0.16} & Deblurred & 0.30 & 1.75 & 0.07 & 0.03 & 0.22 & 0.11 \\
\hline & & & & Original & 4.19 & & 0.09 & & 0.00 & \\
\hline \multirow{2}{*}{ Sinus } & \multirow{2}{*}{0.69} & \multirow{2}{*}{0.49} & \multirow{2}{*}{0.13} & Deblurred & 2.79 & 2.21 & 0.11 & 0.01 & 0.02 & 0.02 \\
\hline & & & & Original & 12.80 & & 0.13 & & 0.00 & \\
\hline \multirow{2}{*}{$\begin{array}{l}\text { Left } \\
\text { zygoma }\end{array}$} & \multirow{2}{*}{0.91} & \multirow{2}{*}{0.85} & \multirow{2}{*}{0.59} & Deblurred & 0.39 & 0.36 & 0.09 & 0.09 & 0.24 & 0.09 \\
\hline & & & & Original & 1.02 & & 0.34 & & 0.09 & \\
\hline \multirow{2}{*}{$\begin{array}{l}\text { Zygomatic } \\
\text { arch }\end{array}$} & \multirow{2}{*}{1.52} & \multirow{2}{*}{0.94} & \multirow{2}{*}{0.51} & Deblurred & 0.38 & 1.68 & 0.18 & 0.08 & 0.10 & 0.07 \\
\hline & & & & Original & 3.71 & & 0.38 & & 0.06 & \\
\hline \multirow{2}{*}{$\begin{array}{l}\text { Right } \\
\text { zygoma }\end{array}$} & \multirow{2}{*}{0.98} & \multirow{2}{*}{0.87} & \multirow{2}{*}{0.47} & Deblurred & 0.29 & 0.41 & 0.10 & 0.07 & 0.21 & 0.09 \\
\hline & & & & Original & 1.25 & & 0.28 & & 0.13 & \\
\hline
\end{tabular}

